تأثير نانو دىاكسيد تيتانيوم و نانو تيوب كربن بر ويزگى هاى جوانهزنى بذر برخى ارقام جو

يوشينهدار (Hordeum vulgare)

\author{
محمودر رضا تدين "،"، محمد رحيمى \\ ' دانشيار گروه زراعت دانشكده كشاورزى دانشگاه شهركرد

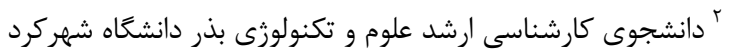 \\ tadayyon-m@agr.sku.ac.ir :
}

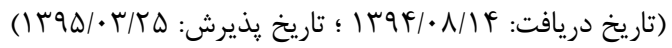

هدف از اين يزوهش، بررسى تأثير نانو ذرات دىاكسيد تيتانيوم (Nano TiO) و نانو تيوب كربنى (CNT)

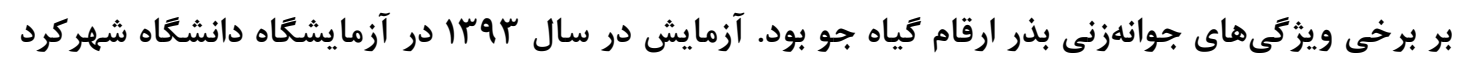

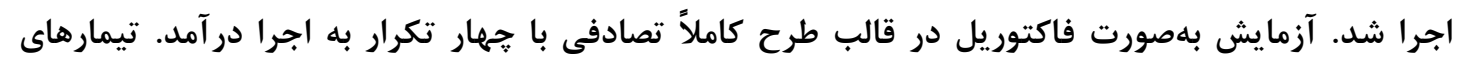

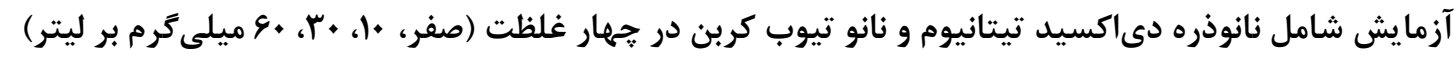

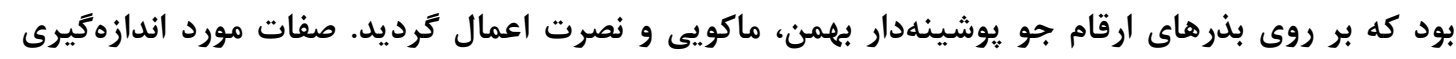

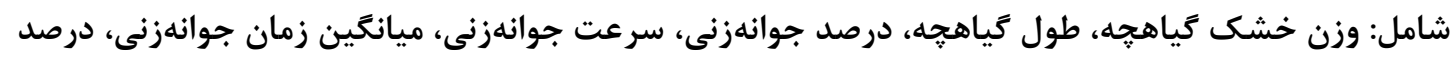

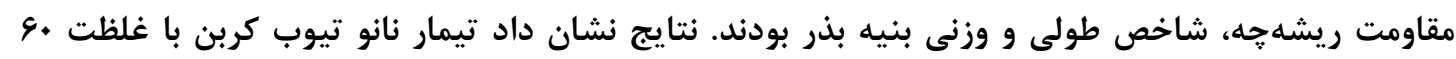
ميلى

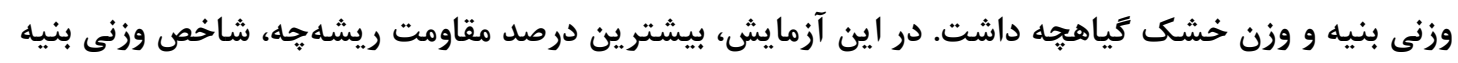

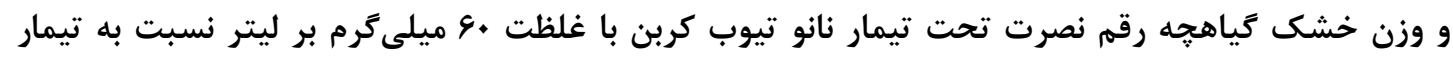

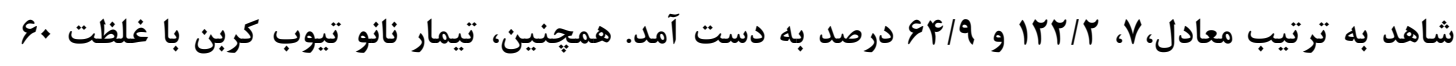
ميلى

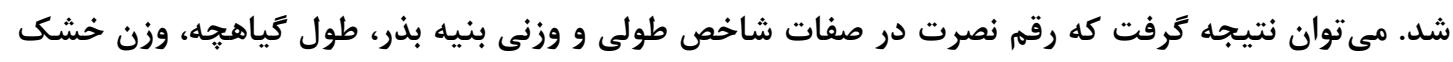

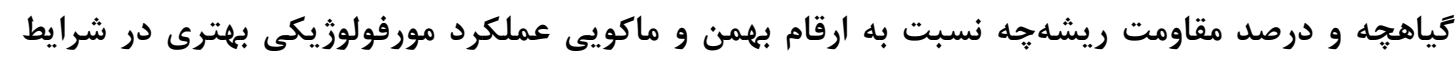
آزمايشعاهى نشان داد.

وازههاى كليدى: بنيه بذر، رقم نصرت، رقم بهمن، وزن تر تياهجه، وزن خشك تياهجه

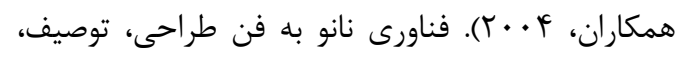
مقدمه توليد و كاربرد ساختار، ابزار و سامانهها در اندازه نانو

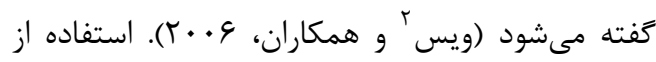

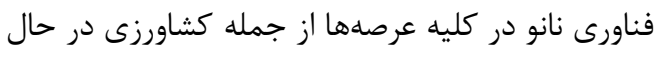

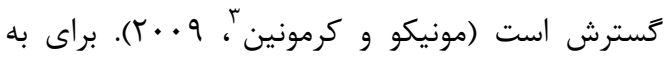

\footnotetext{
${ }^{2}$ Weiss

${ }^{3}$ Monica and Cremonini
}

${ }^{1}$ Akar

جو (Hordeum vulgare) از خانواده كندميان Poaceae كياهان زراعى اهلى شده است (تاجبخش و يورميرزا، זی1ו). جو از لحاظ اهميت بهعنوان زياه زراعى رتبه جهارم يس از گندم، ذرت و برنج را دارا است (آكار' و 


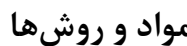

اين آزمايش بهصورت فاكتوريل در قالب طرح كاملاً

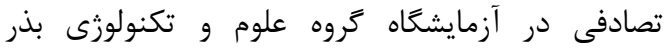

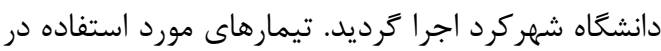

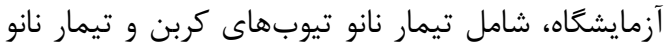
دىاكسيد تيتانيوم در سه غلظت از هر نانوذره شامل:

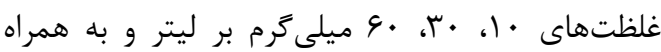
تيمار شاهد و سه رقم جو يوشينهدار و در جهار تكرار بود. علت انتخاب ارقام جو يوشينهدار بررسى اين موضوع

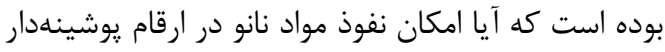

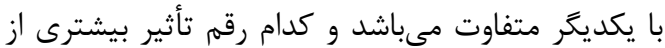
اين موضوع مىيذيرد. در ابتدا بذرهاى جو يوشينهدار

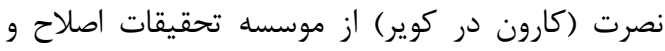

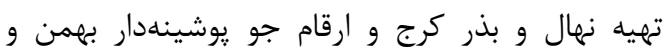

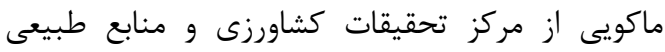
استان مركزى (اراك) تهيه كرديد. ابتدا بذرهاى جو بات

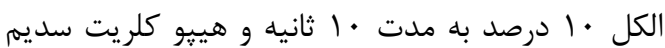
ها درصد تجارى به مدت ب دقيقه ضدعفونى و سيس با آب مقطر شستشو داده شدند. بذرهاى ضدعفونى شده داخل ظروف يترى ديش حاوى كاغذ صافى قرار داده

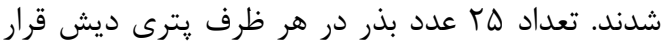

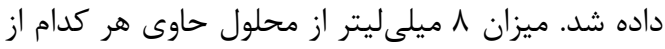
نانو ذرات با غلظتهاى متفاوت بهصورت جدان آدانه، براى آداى

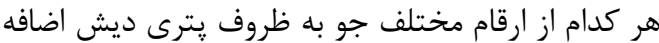

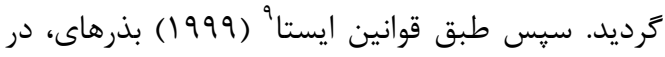

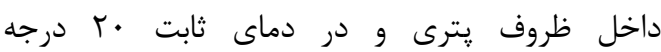
سانتى كراد و به مدت هفت روز در دادرى داخل زرميناتور قرار

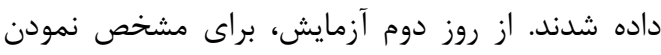

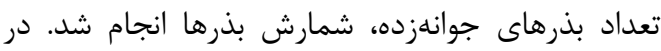

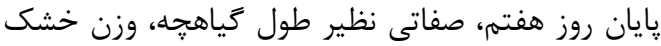
كياهجه با استفاده از خطكش و ترازو اندازهيرى كرديد

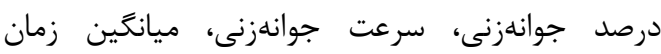

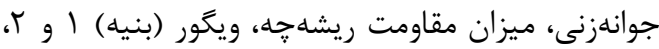
با استفاده از فرمولهاى زير مورد بررسى، اندازهيرى و و

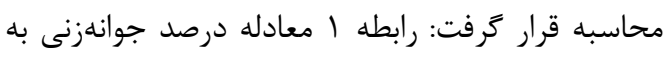
شرح زير مىباشد: كه در آن GP درصد جوانهزنى و

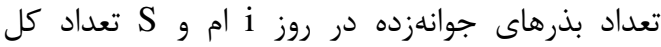

\section{${ }^{9}$ ISTA}

حداقل رساندن تلفات مواد مغذى و افزايش عملكرد كياهان زراعى نياز به مديريت كاربرد مواد نانو مىباشد

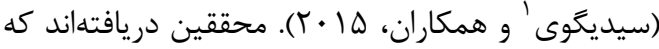
تأثيرات مثبت و منفى نانو ذرات بر رشد و توسعه كياه

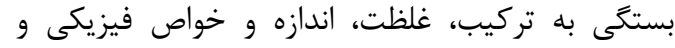

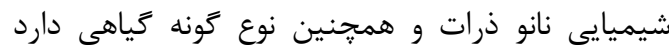

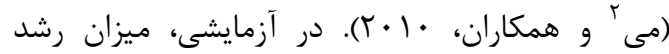
ريشه קه، نسبت به ساقهجه در گَندم تحت تأثير عناصر

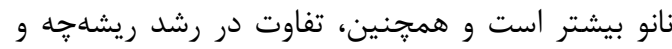
ساقهجه، تحت عناصر نانو ذرات

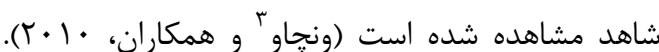

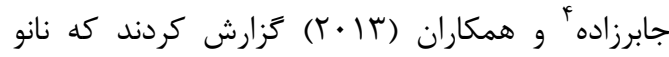

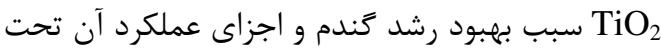
شرايط تنش آبى شده است. نانو تيوب كربنى ماده است كه كربن با قطر يك اتم و به شكل استوانههاى توخالى ساختهشده در سال 1991 توسط ساميوايجيما توليد

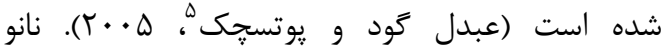
تيوبهاى كربنى، باعث افزايش جوانهزنى بذرها و رشد و

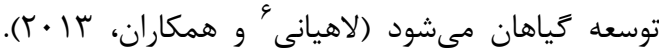

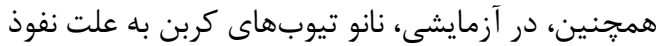
بهتر به غشاى بذر، باعث افزايش جوانهزنى و رشد اوليه

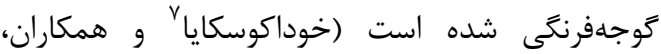

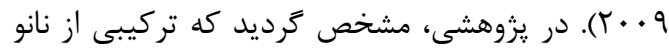
ذرات

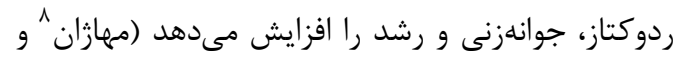

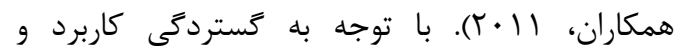
استفاده از مواد نانو در بخش كشاورزى و توليد كياهان و و

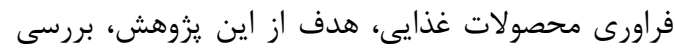

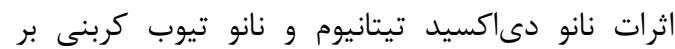
جوانهزنى بذر جند رقم جو بوده است.

\footnotetext{
${ }^{1}$ Siddiqui

${ }^{2} \mathrm{Ma}$

${ }^{3}$ Wenchao

${ }_{5}^{4}$ Jaberzadeh,

${ }^{5}$ Abdel-Goad

${ }^{6}$ Lahiani

${ }^{7}$ Khodakovskaya

${ }^{8}$ Mahajan
} 


\section{نتايج و بحث}

نتايج بهدستآمده از تجزيه واريانس شاخصهاى جوانهزنى در (جدول () نشان داده شده است. اثر متقابل تيمار نانو و ارقام جو بر ميانكَين درصد مقاومت ريشهجه، شاخص طولى و وزنى بنيه بذر، طول و وزن كياهجه در سطح يك درصد و ميانگين زمان جوانهزنى در سطح هانه

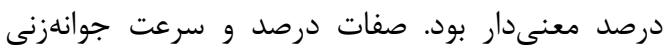
تحت تأثير معنىدار تيمار نانو و ارقام جو قرار كرفت امدات اثر متقابل آنها براى اين صفات معنى دار نكرديد (جدول (). طبق جدول rا، بيشترين درصد جوانهزنى اينى

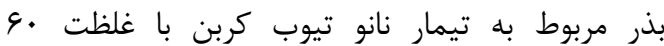

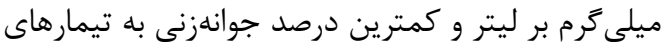

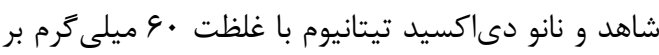
ليتر تعلق داشت. تمامى تيمارهاى اعمالشده نسبت به به بالف شاهد، سبب افزايش سرعت جوانهزنى شدند. طبق نتايج

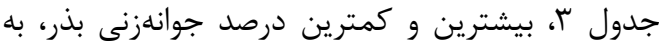

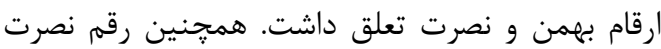
نسبت به ساير ارقام داراى كمترين سرعت جوانهزنى بود. طبق جدول "ا، رقم بهمن داراى بيشترين ميانكين زمان جوانهزنى بذر نسبت به ساير ارقام بود. همجنين بيشترين و كمترين درصد مقاومت ريشه خه بـ به ارقام نصرت و بهمن تعلق داشت (جدول بَ).

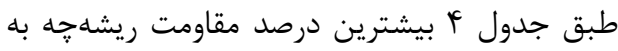

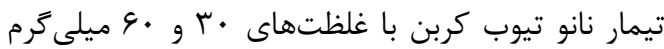

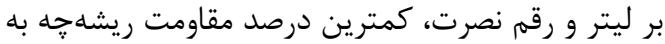

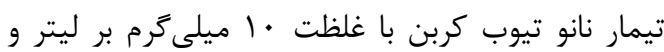

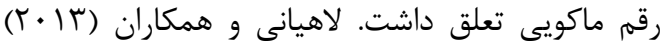
نشان دادند كه بيشترين درصد جوانهزنى بذر كياه جو،

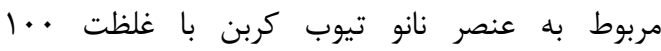

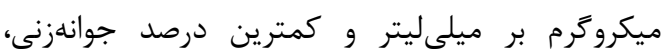
مربوط به تيمار شاهد بوده است.

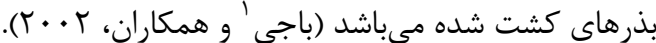

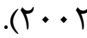

$\mathrm{GP}=(\mathrm{Ni} / \mathrm{S}) \times 100$ رابطه ):

رابطه r معادله سرعت جوانهزنى كه در آن TR سرعت جوانهزنى Ni تعداد بذرهاى جوانهزده در روز موردنظر و Di تعداد روزهاى سيرىشده از شروع تدران

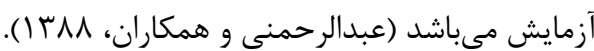
رR $=\Sigma_{1}^{i} \frac{N i}{D i}$

رابطه ب معادله ميانگين زمان جوانهزنى كه در آن ميانگين زمان جوانهزنى و و تعداد بذرهاى

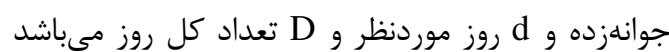

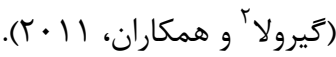

$$
\text { MGT }=\frac{(n 1 \times d 1)+(n 2 \times d 2)+\cdots}{\nu}:{ }^{\prime}
$$

رابطه f معادله شاخص طولى بنيه بذر كه در آن شاخص طولى بنيه بذر، حاصل جمع RL شول بلى ريشهجه و PL طول ساقهجه در GP درصد جوانهزنى مىباشد (مصطفوى و حيدريان، الو (1).). رابطه f رابطه ه معادله شاخص وزنى بنيه بذر كه در آن

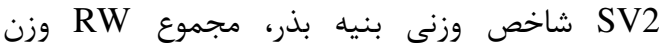

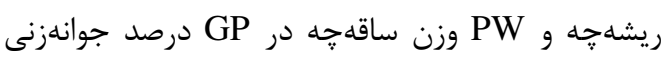
مىباشد (ايستا، 999 1؛ بارانى دستجردى و همكاران،

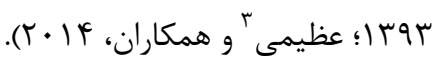
SV2= $(\mathrm{PW}+\mathrm{RW}) \times \mathrm{GP} \quad$ ابطه هان رابطه 9 معادله مقاومت ريشه جه به شرح زير

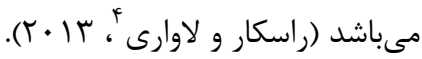

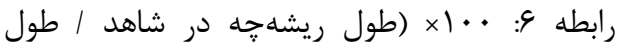

ريشهجه در تيمار )

در اين آزمايش دادهها با نرمافزار SAS و

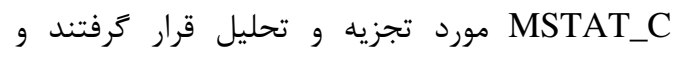
ميانكين دادهها با نرمافزار EXCEL نمايش داده شد. مقايسه ميانكَين دادهها بر اساس آزمون حداقل اختلاف معنى مار (LSD) انجام كرفت.

\footnotetext{
${ }^{1}$ Bajji

${ }^{2}$ Gairola

${ }^{3}$ Azimi

${ }^{4}$ Raskar and Laware
} 
جدول ا- تجزيه واريانس (ميانكين مربعات) درصد جوانهزنى، ميانكين زمان جوانهزنى، سرعت جوانهزنى، درصد مقاومت ريشهجه، طول گياهجه،

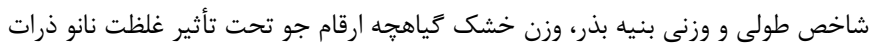

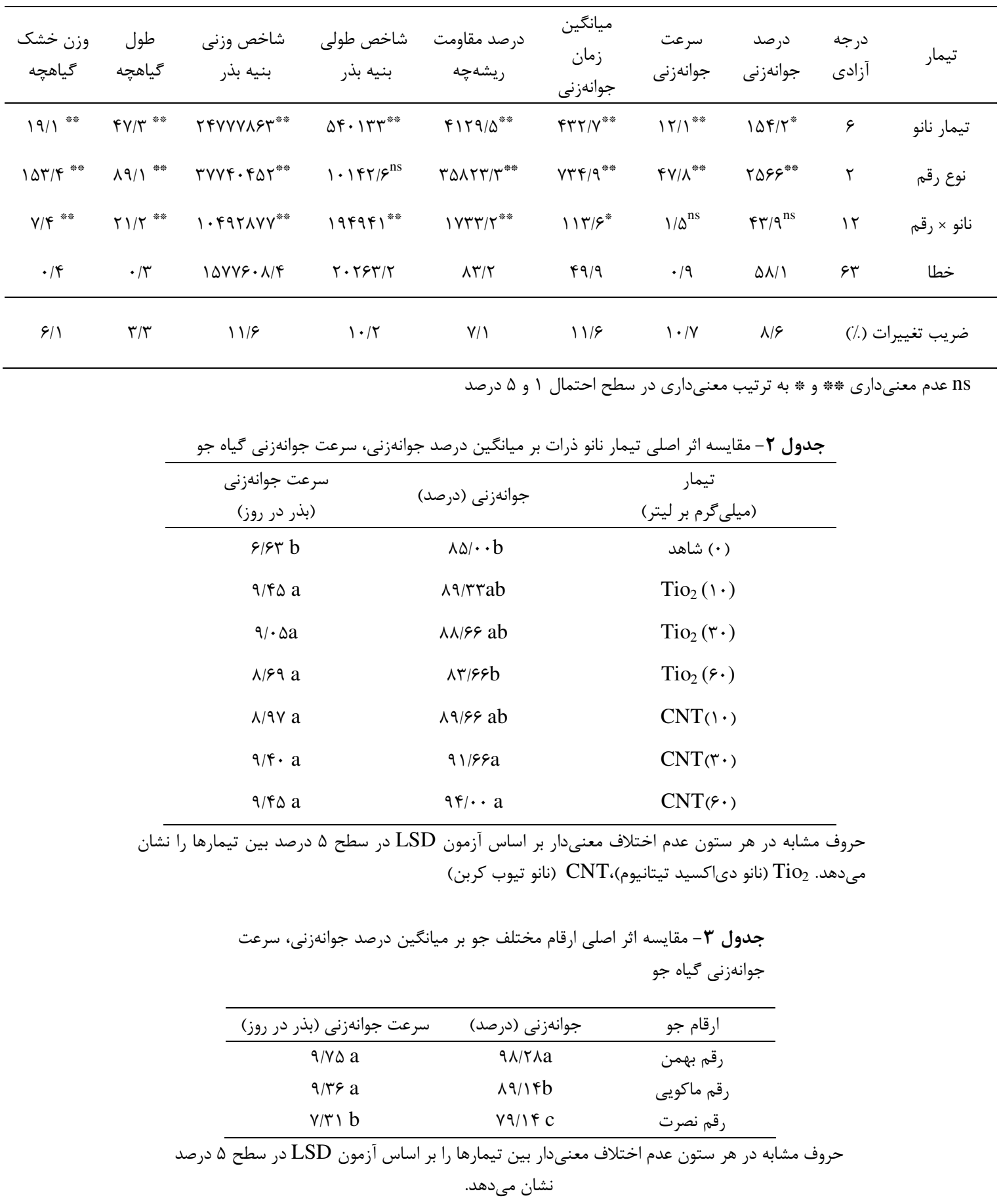


مجله يزوهشهاى بذر ايران/ سال سوم/ شماره دوم/ هوسا

جدول F- Fقايسه اثر متقابل تيمارهاى نانو در ارقام جو براى ميانگين زمان جوانهزنى، درصد مقاومت ريشهجه، طول و وزن خشك گياهجه و بنيه

بذر

\begin{tabular}{|c|c|c|c|c|c|c|c|}
\hline 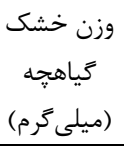 & $\begin{array}{c}\text { كَاهجه } \\
\text { (سانتى متر) } \\
\end{array}$ & وزنى بنيه & شاخص طولى بذيه بذر & مقاومت ريشه جه & جوانهزنين زمان (روز) & جام ام & 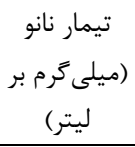 \\
\hline $\mathrm{V} / \wedge \mathrm{V} \mathrm{k}$ & $11 / \Delta \cdot 1$ & qvqf de & $11 \mathrm{rVg}$ & $1 \cdots f g$ & NFa & بهمن & \\
\hline N/qV ij & $10 / 9 \cdot \mathrm{gh}$ & V५९৭f & Iror d-f & $1 . . f g$ & $v r b$ & ماكويى & (•) شاهد \\
\hline $9 / 9 /$ hi & $\mid 1 / 8 \wedge 1$ & $V \cdot r q f$ & $\Delta f \cdot / v h$ & $\cdots f g$ & $q \cdot / \Delta \cdot c-f$ & نصرت & \\
\hline १/१ $\mathrm{h}$ & $191 \wedge 9$ ef & $1 \cdot 11 \cdot \mathrm{de}$ & $19 \Delta \mathrm{rbc}$ & $10 \mathrm{~N} / 9 \mathrm{bc}$ & $\Delta q / \Delta \cdot c-f$ & بهمن & \\
\hline $1 \cdot / 10 \mathrm{gh}$ & $10 / 9 r \mathrm{gh}$ & $1 \cdot \Delta q \cdot c-\mathrm{e}$ & $|\& \lambda| \mathrm{cd}$ & MN/TF gh & $\Delta \Delta / V \Delta$ ef & ماكويى & $\mathrm{Tio}_{2}(1 \cdot)$ \\
\hline$|f /| \cdot b$ & $|N / \Lambda| c$ & Irrg. b & $\mid f \Delta \Lambda \mathrm{cd}$ & $199 / 9 \mathrm{fb}$ & $\Delta s$ ef & نصرت & \\
\hline q/^.hi & $\mid \mathrm{Q} / \mathrm{Argh}$ & $11 f q \cdot b-e$ & $1 \Delta \Delta r \mathrm{~cd}$ & $\mid \Delta N / \Gamma \mathrm{bc}$ & $9 \Delta \mathrm{b}-\mathrm{e}$ & بهمن & \\
\hline $11 / \cdot \Delta \mathrm{fg}$ & $\mid \Delta / \Delta \varphi \mathrm{hi}$ & $\| \Delta q \cdot b-d$ & $1 r \Delta \Delta d-f$ & $\wedge \Delta / \wedge \wedge \mathrm{hi}$ & $\Delta r / \Delta \cdot f$ & ماكويى & $\mathrm{Tio}_{2}(r \cdot)$ \\
\hline $\mid r / \cdot V \mathrm{de}$ & IG/VT ef & $\| \Delta \varepsilon \cdot b-d$ & Iror d-f & $\mid \mathrm{FV} / \cdot \mathrm{cd}$ & $\Delta T / V \Delta \mathrm{f}$ & نصرت & \\
\hline 9/VVhi & $1 \mathrm{Q} / 9 \Delta \mathrm{gh}$ & १९९८ de & $\mid \Delta \& A \mathrm{~cd}$ & $19 T / \pi b$ & $\Delta \Lambda / V \Delta d-f$ & بهمن & \\
\hline $11 / r \Delta$ ef & IV/Tr de & ||$\Delta \mid \cdot b-e$ & $|f| r$ de & $q r / \cdot r \mathrm{gh}$ & $\Delta T / \Delta \cdot f$ & ماكويى & $\mathrm{TiO}_{2}(\xi \cdot)$ \\
\hline Ir/AT cd & $1 \varepsilon / \mathrm{r} \vee \mathrm{fg}$ & $111 r \cdot b-e$ & $111 \cdot f g$ & $19 \cdot / 4 b$ & $\Delta I / T \Delta \mathrm{f}$ & نصرت & \\
\hline G/KA 1 & $1 \cdot / \Delta \wedge \mathrm{m}$ & VVGVf & $1 . \Delta 9 \mathrm{~g}$ & $1.9 / \mathrm{Af}$ & $4 \wedge \mathrm{b}-\mathrm{d}$ & بهمن & \\
\hline$\wedge / \wedge j \mathrm{k}$ & $11 / v \Delta 1$ & qvaq e & $1 \cdot \Delta v g$ & $G F / Y F j$ & $\Delta V$ ef & ماكويى & $\mathrm{CNT}(1 \cdot)$ \\
\hline $\mid r / \cdot r c$ & $|V / 9| d$ & $|r| f \cdot b c$ & $|f| f$ de & $|\xi \xi| \xi \mathrm{b} \mathrm{f}$ & $\Delta N / \Delta \cdot d-f$ & نصرت & \\
\hline $\mathrm{V} / 9 \Delta \mathrm{k}$ & Ir/VYk & $1 \cdot 14 \cdot \mathrm{de}$ & IFFs e-g & $\mid r g / r \mathrm{~d}$ & $q \vee b-d$ & بهمن & \\
\hline $9 / V$ hi & $\mid \psi / \Delta \& \mathrm{j}$ & $1 \cdot 19 \cdot \mathrm{de}$ & $1 r \Delta \Delta d-f$ & $V \& / K r$ ij & $\Delta \cdot / V \Delta \mathrm{f}$ & ماكويى & $\mathrm{CNT}\left(\Gamma^{\top} \cdot\right)$ \\
\hline $1 F / q \cdot b$ & $r . / 99 \mathrm{a}$ & $1 \Delta r \cdots a$ & IVGr ab & $|\wedge r| \cdot a$ & $q \psi / r \Delta b-e$ & نصرت & \\
\hline$\Lambda / r \cdot j \mathrm{k}$ & $\mid F / \wedge \Delta \mathrm{ij}$ & $1 \cdot q v \cdot b-e$ & $|f v| \mathrm{cd}$ & $19 \cdot 1 \mathrm{Ab}$ & sf/VDb-e & بهمن & \\
\hline $\mid r / V V c d$ & $r \cdot / 11 \mathrm{~b}$ & $1 \cdot 19 \cdot b-e$ & $1191 \mathrm{a}$ & $\mid r r / f e$ & $\Delta \vee$ ef & ماكويى & $\mathrm{CNT}(\varphi \cdot)$ \\
\hline $10 / \wedge \Delta \mathrm{a}$ & $r \cdot / V V a b$ & $1099 \cdot a$ & $1 \wedge+9 a b$ & $|\wedge \mathrm{V}| \cdot \mathrm{Fa}$ & $99 \mathrm{bc}$ & نصرت & \\
\hline
\end{tabular}

حروف مشابه در هر ستون عدم اختلاف معنىدار بر اساس آزمون LSD در سطح ه درصد بين تيمارها را نشان مىدهد. تيتانيوم)،CNT (نانو تيوب كربن)

طولى بنيه بذر به ترتيب به تيمار نانو تيوب كربن با غلظت •و ميلى و تيمار شاهد و رقم جو يوشينهدار نصرت تعلق داشت. بيشترين مقدار شاخص وزنى بنيه بذر و وزن خشك

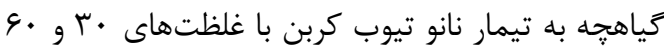
ميلى بنيه بذر r، به تيمار شاهد و ارقام جو يوشينهدار نصرت

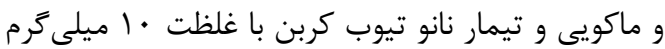
بر ليتر و رقم جو يوشينهدار بهمن تعلق دارد. بيشترين

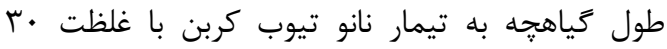
ميلى

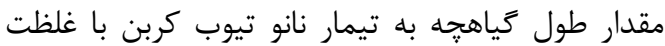

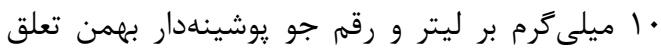

تأثير مثبت نانو تيوب كربن نسبت به شاهد بر بذر خياه ذرت نشان داده شده است. نانو تيوب كربن در

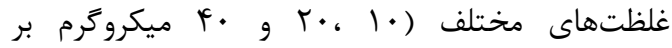

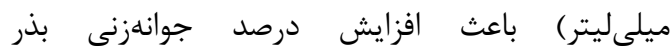
Fوجهفرنكى نسبت به شاهد شده است (خوداكووسكايا و باعت

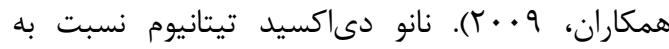
شاهد، سبب افزايش درصد جوانهزنى و سرعت جوانهزنى و كاهش ميانگين زمان جوانهزنى بذرهاى مريمَلى شده

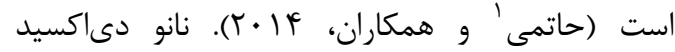
تيتانيوم در غلظت · f ميكروگرم بر ميلىليتر سبب

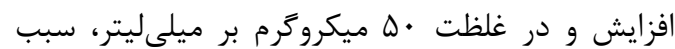
كاهش درصد جوانهزنى گياه يیاز شد (راسكار و لاوار، r| •r). طبق جدول ؟ا، بيشترين و كمترين شاخص

${ }^{1}$ Hatami 


\begin{tabular}{|c|c|}
\hline 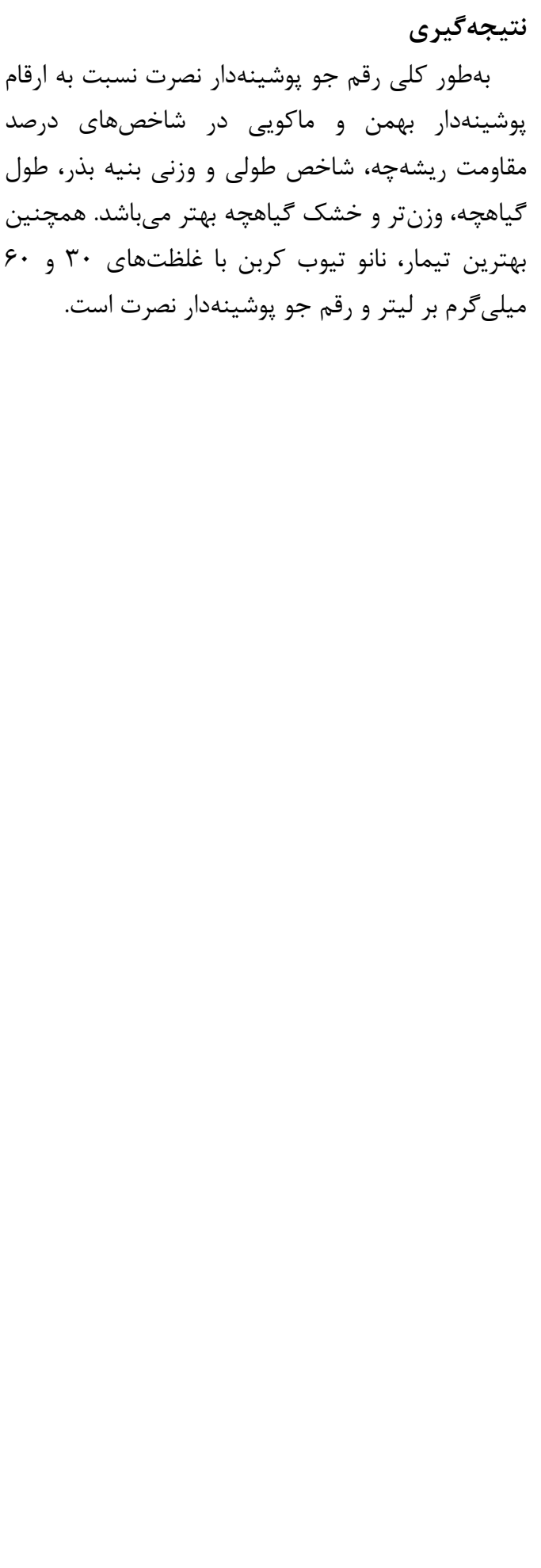 & مان \\
\hline & $\begin{array}{l} \\
{ }^{1} \text { Yang and Watts } \\
{ }^{2} \text { Mahmoodzadeh } \\
{ }^{3} \text { Adhikari } \\
{ }^{4} \text { Buzea } \\
{ }^{5} \text { Mahmoodzadeh,and Aghili }\end{array}$ \\
\hline
\end{tabular}




$$
\begin{aligned}
& \text { منابع } \\
& \text { بارانى دستجردى، م. رفيع الحسينى، م. و دانش شهركى، ع.ا. بوسا. تأثير تنش خشكى و محلولياشى روى و منحَنز بر }
\end{aligned}
$$

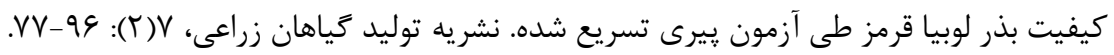

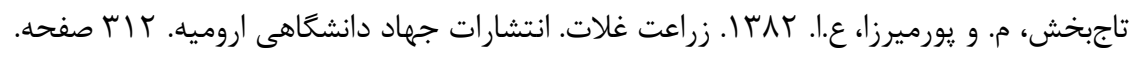

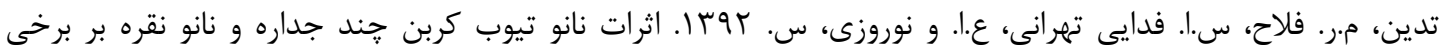

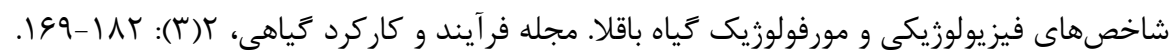

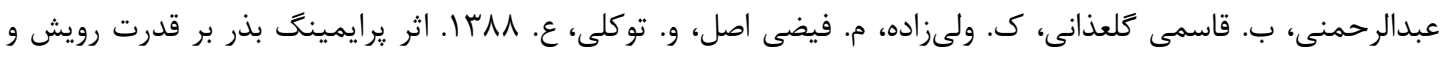

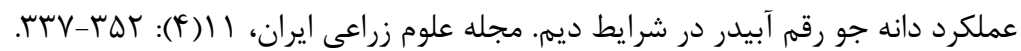

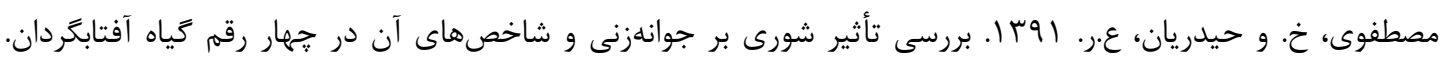

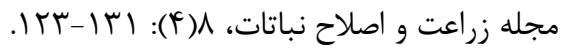

Abdel-Goad, M., and Potschke, P. 2005. Phenological characterization of melt processed poly carbonate multi walled carbon nanotube composite. Journal Non-Newtonian Fluid Mechanics, 128(1): 2-6.

Adhikari, T. Kundu, S., and Subba Rao, A. 2013. Impact of $\mathrm{SiO}_{2}$ and Mo Nano particles on seed germination of rice (Oryza sativa L.). International Journal of Agriculture and Food Science Technology, 4: 809-816.

Akar, T. Avci, M., and Dusunceli, F. 2004. Barley: Post-harvest operations. The Central Research Institute for Field Crops, Ankara, Turkey.

Azimi, R. Jankju Borzelabad, M. Feizi, H., and Azimi, A. 2014. Interaction of $\mathrm{SiO}_{2}$ nanoparticles with seed prechilling on germination and early seedling growth of tall wheatgrass Agropyron elongatum. Polish Journal of Chemical Technology, 16(3): 25-29.

Bajji, M. Kinet, J.M., and Lutts, S. 2002. Osmotic and ionic effects of $\mathrm{NaCl}$ on germination, early seedling growth, and ion content of Atriplex halimus Chenopodiaceae. Canadian Journal of Botany, 80(3): 297-304.

Buzea, C. Pacheco Blandino, I., and Robbie, K. 2007. Nanomaterials and nanoparticles: sources and toxicity. Biointerphases, 2(4): 17-71.

Gairola, K.C. Nautiyal, A.R., and Dwivedi, A.K. 2011. Effect of temperatures and germination media on seed germination of Jatropha curcas L. Advances in Bioresearch, 2(2): 66-71.

Hatami, M. Ghorbanpour, M., and Salehiarjomand, H. 2014. Nano-anatase $\mathrm{TiO}_{2}$ modulates the germination behavior and seedling vigority of some commercially important medicinal and aromatic plants. Journal Biological and Environmental Science, 8(22): 53-59.

ISTA. 1999. International rules for seed testing. seed science and technology, 27, Supplement.

Jaberzadeh, A. Moaveni, P. Tohidi Moghadamh, R., and Zahedi, H. 2013. Influence of bulk and nanoparticles titanium foliar application on some agronomic traits, seed gluten and starch contents of wheat subjected to water deficit stress. Notulae Botanicae Horti Agrobotanici ClujNapoca, 41(1): 201-207.

Khodakovskaya, M., Dervishi, E., Mahmood, M., Xu, Y., Li, Z., Watanabe, F., and Biris, A.S. 2009. Carbon nanotubes are able to penetrate plant seed coat and dramtically affect seed germination and plant growth. American Chemical Society, 3(10): 3221-3227.

Lahiani, M.H. Dervishi, E., Chen, J., Nima, Z., Gaume, A., Biris, A.S., and Khodakovskaya, M.V. 2013. Impact of carbon nanotube exposure to seeds of valuable crops. ACS Applied Materials Interfaces, 5(16): 7965-7973. 
Ma, X., Geiser-Lee, J., Deng, Y., and Kolmakov, A. 2010. Interactions between engineered nanoparticles (ENPs) and plants: phytotoxicity, uptake and accumulation. Science of the Total Environment, 408(16): 3053-3061.

Mahajan P., Dhoke S.K., and Khanna A.S. 2011. Effect of Nano-ZnO particle suspension on growth of mung (Vigna radiate) and gram (Cicer arietinum) seedlings using plant agar method. Hindawi Publishing Corporation Journal of Nanotechnology, 1-7.

Mahmoodzadeh, H., Nabavi, M., and Kashefi, H. 2013. Effect of nanoscale titanium dioxide particles on the germination and growth of canola (Brassica napus). Journal of Ornamental and Horticultural Plants, 3(1): 25-32.

Mahmoodzadeh, H., and Aghili, R. 2014. Effect on germination and early growth characteristics in wheat plants (Triticum aestivum L.) seeds exposed to $\mathrm{TiO}_{2}$ nanoparticles. Journal of Chemical Health Risks, 4(1): 29-36.

Monica, R.C., and Cremonini, R. 2009. Nanoparticles and higher plants, Caryologia, 62(2): 161165.

Raskar, S.V., and Laware, S.L. 2013. Effect of titanium dioxide nano particles on seed germination and germination indicws in onion. Plant Sciences Feed, 3(7): 103-107.

Siddiqui, M.H., Al-Whaibi, M.H., Firoz, M., and Al-Khaishany, M.Y. 2015. Role of nanoparticles in plants. In nanotechnology and plant sciences. Springer International Publishing, 19-35.

Weiss, J., Takhistov, P., and McClements, D.J. 2006. Functional materials in food nanotechnology. Journal of Food Science, 71(9): 107-116.

Wenchao, Du., Yuanyuan, S., Rong, Ji., Jianguo, Zhu., Jichum, Wu., and Hongyan, Guo. 2010. $\mathrm{TiO}_{2}$ and $\mathrm{ZnO}$ nanoparticles negatively affect wheat growth and soil enzyme activities in agricultural soil. Journal of Environmental Monitoring, 13(4): 822-828.

Yang, L., and Watts, D.J. 2005. Particle surface characteristics may play an important role in phytotoxicity of alumina nanoparticles. Toxicology Letters, 158(2): 122-132. 


\title{
Effect of Titanium Dioxide Nanoparticles and Carbon Nanotube on Seed Germination Characteristics of Some Hordeum vulgare Hulled Varieties
}

\author{
Mahmoud Reza Tadayon ${ }^{1,}$,, Mohammad Rahimi ${ }^{2}$ \\ ${ }^{I}$ Associate Professor of Seed Science and Technology of Shahrekord University, Shahrekord, Iran \\ ${ }^{2}$ M.Sc. Student of Seed Science and Technology of Shahrekord University, Shahrekord, Iran \\ "Corresponding author, E-mail adders: tadayyon-m@agr.sku.ac.ir
}

(Received: 05.11.2015 ; Accepted: 14.06.2016)

\begin{abstract}
The purpose of this study was to evaluate the effect of $\mathrm{Nano}^{\mathrm{TiO}}{ }_{2}$ and Nano CNT on some germination indices and growth parameters of some hulled barley cultivars. The experiment was conducted in a laboratory at Shahrekord University during 2014. The study was a factorial, adopting a completely randomized design with four replications. Treatments consisted of titanium dioxide nanoparticles and carbon nanotube (CNT) in four concentrations $\left(0,10,30\right.$ and $\left.60 \mathrm{mg} . \mathrm{l}^{-1}\right)$ which were applied to seeds of hulled barley cultivars such as Bahman, Makoii and Nosrat. The traits measured were dry weight of seedling and seedling length, germination percentage, germination rate, mean germination time, the percentage of resistance of radical, seedling vigor length and weight index. The results showed that carbon nanotubes treatments with $60 \mathrm{mg} . \mathrm{l}^{-1}$ had a significant impact on germination percentage, germination rate, seedling vigor length and weight index and seedling dry weight, as compared with other treatments. In this experiment, the highest percentages of resistance of radicle, seedling vigor weight index and seedling dry weight of Nosrat Cultivar were obtained under $60 \mathrm{mg} \cdot \mathrm{l}^{-1}$ carbon nanotube treatment, which were $70,122.2$ and $64.9 \%$, respectively, as compared with control treatment. In addition, $60 \mathrm{mg} . \mathrm{l}^{-1}$ carbon nanotube treatment increased the seedling vigor of Makoii cultivar by $39.8 \%$, as compared with the control treatment. The findings were that in terms of seedling vigor length and weight index, seedling length, seedling dry weight and the percentage of resistance of radical, the Nosrat Cultivar showed better morphological characteristics than Bahman and Makoii cultivars, under laboratory conditions.
\end{abstract}

Keywords: Seedling vigor, Nosrat cultivar, Bahman cultivar, Seedling fresh weight, Seedling dry weight 